\title{
An FFT-based signal identification approach for obtaining the propagation constants of the leaky modes in layered media
}

\author{
Luc Knockaert*, Hendrik Rogier, Daniël De Zutter \\ INTEC-IMEC, Ghent University, St-Pietersnieuwstraat 41, B-9000 Ghent, Belgium
}

Received 5 May 2004; received in revised form 8 July 2004

\begin{abstract}
We propose to find the propagation constants of modes in layered media by means of signal identification methods. To this effect we employ Cauchy's theorem, conformal mapping and Fast Fourier Transform (FFT) techniques to generate relevant Hankel moments, afterwards to be processed with selected signal identification algorithms. The method, terminated by a few Newton steps, provides a batch of highly accurate roots in appropriate disks or half-disks.
\end{abstract}

(c) 2004 Elsevier GmbH. All rights reserved.

Keywords: Electromagnetic propagation in stratified media; Leaky waves; Signal resolution; Fast Fourier transforms; Zeros

\section{Introduction}

Leaky waves, which are found as complex solutions of the dispersion relation for dielectric waveguides, play an important role in many electromagnetic (EM) analysis tools [1-3] as well as in practical devices such as leaky wave antennas [4]. Locating the complex zeros, however, can be quite cumbersome, especially when the dielectric substrate consists of a number of different dielectric layers. In this paper, we propose techniques based on signal identification algorithms to efficiently and accurately locate the complex zeros of dispersion relations for layered media. The techniques can be applied for layered substrates consisting of an arbitrary number of layers. The top and bottom substrate can either be open or, for numerical reasons, terminated by a perfectly matched layer (PML) [5-7].

Signal identification of exponential sum models (ESM) is a frequently occurring and recurrent topic in signal processing. The reason for this is that many physical signals, from time series in medicine and economics to spectral analysis in astronomy and sonar applications $[8,9]$ can be expressed

\footnotetext{
* Corresponding author. Tel.: +3292643328; fax: +3292643593

E-mail address: knokaert@intec.UGent.be (L. Knockaert).
}

as sums of damped exponentials. Moreover, ESM has also been used in EM analysis techniques, e.g. in the complex image method for determining the Green's function in layered media. More mathematically speaking, it can be proved that large classes of signals can be expressed as infinite sums of exponentials, due to their $L_{p}$ completeness over selected intervals $[10,11]$. In addition, modelling by exponential sums is inherent in linear systems theory [12] and its underlying Hankel matrix framework [13,14]. Lastly and rather unexpectedly, exponential signal identification has recently been used for reconstructing polygonal shapes from geometrical moments [15].

In this paper, we propose to find the roots of transcendental equations by means of signal identification techniques. As a generalization of methods in [16,17], we employ Cauchy's theorem, conformal mapping and FFT techniques to generate the relevant Hankel moments, which are afterwards processed with selected signal identification algorithms. Our choice (not exhaustive) is one of the four following algorithms: the Pencil-of-Function method [18], the SVD rank-based method [9], the Prony-Burrus-Parks method $[19,20]$ and a Neville-type interpolation method [21]. Applied to finding the propagation constants of the 
leaky modes in stratified media, which correspond to the complex zeros of transcendental dispersion relations, the method, terminated by a few Newton steps, provides a batch of highly accurate roots in selected disks or half-disks.

\section{Roots of transcendental equations}

It is well-known [16,17] that, given a function $F(z)$ which is analytic in a simply connected open domain $\Omega$ bounded by a simple Jordan curve $\Gamma$, except for a simple pole at $p_{0}$, we can recover this pole by means of the formula

$p_{0}=\frac{\oint_{\Gamma} z F(z) \mathrm{d} z}{\oint_{\Gamma} F(z) \mathrm{d} z}$.

This follows at once from Cauchy's theorem. For an analytic function $f(z)$ exhibiting a single simple zero $z_{0}$ in $\Omega$ we can take $F(z)=1 / f(z)$ in order to find $z_{0}$ by formula (1). Of course, one must be sure that only one zero is present in $\Omega$. This can be tested by making use of the principle of the argument [22], which states that for an analytic function $f(z)$ with $m$ zeros inside $\Omega$ we can write

$m=\frac{1}{2 \pi i} \oint_{\Gamma} \frac{f^{\prime}(z)}{f(z)} \mathrm{d} z$.

When $m=1$, formula (1) will yield the unique zero. In order to generalize the approach to more than one zero we need the following [17].

Theorem 1. Let the analytic function $f(z)$ have exactly $m$ simple zeros $z_{1}, z_{2}, \ldots, z_{m}$ in $\Omega$ and let $g(z)$ be analytic such that $g\left(z_{k}\right) \neq 0$ for $k=1,2, \ldots, m$. Then there exist non-vanishing coefficients $\tilde{d}_{k}, k=1,2, \ldots, m$ such that the Hankel moments

$$
\begin{aligned}
h_{n} & =\frac{1}{2 \pi i} \oint_{\Gamma} \frac{g(z)}{f(z)} z^{n} \mathrm{~d} z \\
& =\sum_{k=1}^{m} \tilde{d}_{k} z_{k}^{n}, \quad n=0,1,2, \ldots .
\end{aligned}
$$

Proof. The function $f(z)$ can be written as

$$
f(z)=\frac{\prod_{k=1}^{m}\left(z-z_{k}\right)}{r(z)},
$$

where $r(z)$ is analytic in $\Omega$ with $r(z) \neq 0$. Since

$$
\frac{1}{\prod_{k=1}^{m}\left(z-z_{k}\right)}=\sum_{k=1}^{m} \frac{c_{k}}{z-z_{k}}
$$

with

$c_{k}=\frac{1}{\prod_{l \neq k}\left(z_{k}-z_{l}\right)}$, application of Cauchy's theorem yields

$h_{n}=\frac{1}{2 \pi i} \oint_{\Gamma} \frac{g(z)}{f(z)} z^{n} \mathrm{~d} z=\sum_{k=1}^{m} c_{k} g\left(z_{k}\right) r\left(z_{k}\right) z_{k}^{n}$

with $n=0,1,2, \ldots$. This completes the proof.

Note that a straightforward choice for the function $g(z)$ is the constant function $g(z)=1$. The other straightforward choice $g(z)=f^{\prime}(z)$, with $n=0$, will be employed mainly to determine the number of zeros $m$.

We will restrict ourselves to zeros in $\mathscr{D}$, the open unit disk for the following reason: consider the conformal mapping $\eta(u)$ from $\mathscr{D}$ onto $\Omega$. This mapping always exists, by virtue of the Riemann mapping theorem [23]. Then the zeros of $f(z)$ in $\Omega$ correspond with the zeros of $f(\eta(u))$ in $\mathscr{D}$. Taking $g(u)=1$, the Hankel moments $h_{n}$ can be written as

$h_{n}=\frac{1}{2 \pi} \int_{0}^{2 \pi} \frac{\mathrm{e}^{i(n+1) \theta}}{f\left(\eta\left(\mathrm{e}^{i \theta}\right)\right)} \mathrm{d} \theta=\sum_{k=1}^{m} d_{k} u_{k}^{n}$

with $n=0,1,2, \ldots$. Opting for the $(N+1)$-point closed trapezoidal quadrature rule we obtain

$h_{n}=\frac{1}{N} \sum_{k=0}^{N-1} \frac{\mathrm{e}^{2 \pi i(n+1) k / N}}{f\left(\eta\left(\mathrm{e}^{2 \pi i k / N}\right)\right)}+\varepsilon_{n}$,

where $\varepsilon_{n}$ is the error associated with the quadrature rule. Assuming that the error terms are sufficiently small—see the appendix for some pertinent error bounds-and choosing $N$ as a power of two, (8) is most effectively calculated by means of an FFT. After obtaining the Hankel moments, and processing them with one or more of the signal identification algorithms of the next section, we finally obtain the unit disk zeros $u_{k}$ and the $\Omega$-domain zeros by $z_{k}=\eta\left(u_{k}\right)$.

\section{Signal identification algorithms}

Given the Hankel moments

$h_{n}=\sum_{k=1}^{m} d_{k} u_{k}^{n}, \quad n=0,1,2, \ldots$,

the zeros $u_{k}$ are recovered by judiciously processing the moments $h_{n}$ by means of one of the following four algorithms. Note that there exist other algorithms, such as the ones in $[13,24]$, but we have to be restrictive somehow, and therefore we limit ourselves to the non-exhaustive but representative list below.

\subsection{Algorithm 1: Pencil-of-Function method}

Consider the $m \times m$ Hankel matrix

$H_{m}=\left(\begin{array}{ccc}h_{0} & \cdots & h_{m-1} \\ \vdots & \cdots & \vdots \\ h_{m-1} & \cdots & h_{2 m-2}\end{array}\right)$ 
and its shifted version

$H_{m}^{1}=\left(\begin{array}{ccc}h_{1} & \cdots & h_{m} \\ \vdots & \cdots & \vdots \\ h_{m} & \cdots & h_{2 m-1}\end{array}\right)$.

It is then easy to prove $[17,18]$ that the zeros $u_{k}$ are the eigenvalues of the generalized eigenvalue problem

$H_{m}^{1}=\lambda H_{m}$.

In this method, we need the first $M=2 m$ Hankel moments $h_{0}, \ldots, h_{M-1}$.

\subsection{Algorithm 2: SVD rank-based method}

The SVD-based method [9] exploits the fact that the Hankel matrix $H_{m+1}$ exhibits a unit rank deficit, i.e. it has rank exactly $m$. More generally, we can say that $H_{m+r}$ has rank exactly $m$, for all $r \geqslant 0$. Hence, the nullspace of $H_{m+1}$ is the span of a unit norm vector $c$ representing the coefficients of the root polynomial of the zeros $u_{k}$. Taking $U \Sigma V^{H}$ as the SVD of $H_{m+1}$, finding this nullspace is equivalent with solving $U \Sigma V^{H} c=0$, or since $U$ is unitary $\Sigma V^{H} c=0$. If the singular values are sorted in decreasing order, we have $\Sigma_{1} \geqslant \Sigma_{2} \cdots \geqslant \Sigma_{m}>\Sigma_{m+1}=0$, from which we conclude that $c$ is the last column of the right singular factor $V$. Note that the vector $c$ can also be obtained via the minimization procedure

$c=\arg \min _{x} \frac{\left\|H_{m+1} x\right\|}{\|x\|}$,

where $\|\cdot\|$ is the Euclidian norm.

In this method, we need the first $M+1$ Hankel moments $h_{0}, \ldots, h_{M}$.

\subsection{Algorithm 3: Prony-Burrus-Parks method}

Since the Hankel moments $h_{n}$ can be considered as the time-domain impulse response of an IIR filter with $m$ poles and $m-1$ zeros, we can use the Prony-Burrus-Parks algorithm [19] e.g. as implemented in the MATLAB $®$ Signal Processing Toolbox [20].

The function $[$ num, den $]=\operatorname{prony}(\mathrm{h}, \mathrm{m}-1, \mathrm{~m})$ yields the numerator and denominator of the IIR filter and the $u_{k}$ follow from $u=\operatorname{roots}($ den). In this method, we minimally need the first $M$ Hankel moments $h_{0}, \ldots, h_{M-1}$.

\subsection{Algorithm 4: Neville-type interpolation}

Here, we need the first $M$ Hankel moments $h_{0}, \ldots, h_{M-1}$. Supposing we have $M$ different points $w_{1}, w_{2}, \ldots, w_{M}$ not in $\mathscr{D}$ at our disposal, consider the $M$ polynomials of $(M-1)$-th degree defined as

$\sum_{l=1}^{M} K_{k, l} z^{l-1}=\frac{K(z)}{z-w_{k}}, \quad k=1, \ldots, M$, where $K(z)$ is the root polynomial of the points $w_{k}$, i.e.

$K(z)=\prod_{k=1}^{M}\left(z-w_{k}\right)$

Transforming the Hankel moments by means of the $K_{k, l}$ matrix yields

$\hat{h}_{k-1}=\sum_{l=1}^{M} K_{k, l} h_{l-1}=\sum_{l=1}^{m} d_{l} \frac{K\left(u_{l}\right)}{u_{l}-w_{k}}=\sum_{l=1}^{m} \frac{e_{l}}{w_{k}-u_{l}}$

with $e_{l}=d_{l} K\left(u_{l}\right)$. Hence, the transformed Hankel moments $\hat{h}_{0}, \ldots, \hat{h}_{M-1}$ represent the rational function

$V(z)=\sum_{l=1}^{m} \frac{e_{l}}{z-u_{l}}$

sampled at the points $w_{k}$. A fast recursive algorithm to reconstruct the denominator of degree $m$ and numerator of degree $m-1$ of a rational function, given its tabulated values at $M=2 m$ points, is available in [21]. It is constructed as follows: consider the array $v=\left\{v_{1}, v_{2}, \ldots, v_{M}\right\}$ related to the different complex points $\left\{w_{1}, w_{2}, \ldots, w_{M}\right\}$. Then, a rational function $V(z)=a_{M}(z) / b_{M}(z)$ with $m$ poles and $m-1$ zeros such that $V\left(w_{k}\right)=v_{k}$ can be recursively created by the polynomial Neville-type algorithm

$a_{k}(z)=\sigma_{k} a_{k-1}(z)+\left(z-w_{k-1}\right) a_{k-2}(z)$,

$b_{k}(z)=\sigma_{k} b_{k-1}(z)+\left(z-w_{k-1}\right) b_{k-2}(z)$

with initial values $a_{0}=0, a_{1}=v_{1}, b_{1}=b_{0}=1$. The value for $\sigma_{k}$ is found by requiring that $v_{k}=a_{k}\left(w_{k}\right) / b_{k}\left(w_{k}\right)$, i.e.

$\sigma_{k}=\left(w_{k-1}-w_{k}\right) \frac{v_{k} b_{k-2}\left(w_{k}\right)-a_{k-2}\left(w_{k}\right)}{v_{k} b_{k-1}\left(w_{k}\right)-a_{k-1}\left(w_{k}\right)}$.

In our case, we naturally take $v_{k}=\hat{h}_{k-1}$. To implement the method we still have leeway in choosing the points $w_{k}$. In our case we take the $M$-th roots of unity which reside on the unit circle, hence not inside the unit disk. We have $w_{k}=W^{k-1}$, with $W=\mathrm{e}^{2 \pi i / M}$. The corresponding transformation matrix is

$K_{k, l}=W^{-(k-1) l}$

implying that the transform $\hat{h}_{k-1}=\sum_{l=1}^{M} K_{k, l} h_{l-1}$ can readily be implemented by means of the DFT. Finally, the zeros $u_{k}$ are found as the poles of $V(z)$, i.e. the zeros of $b_{M}(z)$.

\section{Conformal mappings}

The conformal mapping technique can be used to transform the unit disk, in which the zeros are recovered, to a different region more suitable for our needs. Of particular interest is the mapping

$\eta_{1 / 2}(u)=\frac{u-1+\sqrt{2+2 u^{2}}}{u+1}$, 
which transforms $\mathscr{D}$ into the open halfdisk $\mathscr{D}_{1 / 2}$ defined as $\{u:|u|<1, \mathfrak{R}(u)>0\}$. The mapping $\eta(u)=a \eta_{1 / 2}(u)+b$ amounts to a translation, a rotation and a scaling of the halfdisk.

Another useful mapping is the one transforming the unit disk onto a regular $n$-gon inscribed in the unit circle. It is given by [22]

$$
\begin{aligned}
\eta_{n}(u) & =C \int_{0}^{u}\left(1-w^{n}\right)^{-\frac{2}{n}} \mathrm{~d} w \\
& =C u_{2} F_{1}\left(\frac{1}{n}, \frac{2}{n} ; 1+\frac{1}{n} ; u^{n}\right), \quad n>2,
\end{aligned}
$$

where $C$ is the constant yielding $\eta_{n}(1)=1$ and ${ }_{2} F_{1}(\cdots)$ is the hypergeometric function

${ }_{2} F_{1}(a, b ; c ; z)=\sum_{k=0}^{\infty} \frac{(a)_{k}(b)_{k}}{(c)_{k}} \frac{z^{k}}{k !}$

and $(a)_{k}=a(a+1) \cdots(a+k-1)$ is the Pochhammer symbol.

In general it is well-known that it is sufficient to know the boundary correspondence function, i.e. the mapping between each boundary point of the unit circle and the boundary $\Gamma$. For instance, when $\Gamma$ is given in polar co-ordinates

$\Gamma: z=z(\psi)=\rho(\psi) \mathrm{e}^{i \psi}, \quad 0 \leqslant \psi \leqslant 2 \pi, \quad \rho(\psi)>0$

then the boundary correspondence function is given by $\psi(\theta)$ which satisfies Theodorsen's integral equation [23]

$$
\begin{aligned}
& \psi(\theta)-\theta \\
& =\frac{1}{2 \pi} \mathrm{PV} \int_{0}^{2 \pi} \cot \left(\frac{\theta-\tau}{2}\right) \log (\rho(\psi(\tau))) \mathrm{d} \tau
\end{aligned}
$$

for $0 \leqslant \theta \leqslant 2 \pi$, where PV stands for the Cauchy Principal Value. The resulting conformal mapping is

$\eta(u)=u \mathrm{e}^{\xi(u)}$,

where $\xi(u)$ is

$\xi\left(\mathrm{e}^{i \theta}\right)=\log (\rho(\psi(\theta)))+i[\psi(\theta)-\theta]$

on the unit circle and

$\xi(u)=\frac{1}{2 \pi i} \int_{0}^{2 \pi} \frac{\xi\left(\mathrm{e}^{i \theta}\right)}{\mathrm{e}^{i \theta}-u} \mathrm{de}^{i \theta}$

inside the unit disk. Note that Theodorsen's integral equation is highly non-linear, and therefore, in our context, of limited utility. Also, since too many complicated function evaluations have a serious slowing down effect on the overall processing time, we will not present examples for the regular $n$-gon and restrict ourselves to roots in $\mathscr{D}$ and $\mathscr{D}_{1 / 2}$.

\section{Application to modes in layered media}

\subsection{Dispersion relation}

Consider a planar layered medium consisting of $N$ dielectric layers of infinite horizontal extent with thickness $d_{i}$ and

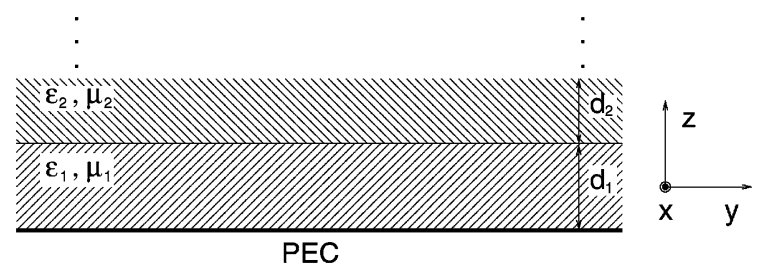

Fig. 1. A layered medium.

permittivity $\varepsilon_{i}, i=1, \ldots, N$, as shown in Fig. 1 . The first and the last layers can either be open or terminated by a perfectly electrical conducting (PEC) plane. In the latter case, one or both of these layers could be PMLs, so that the closed configuration behaves approximately as an open structure. The technique to find the complex propagation constants $\beta$ of the modes that exist in these structures is well-known $[25,26]$ and we will only give a brief overview here. With each layer $i$, we associate a transfer matrix $T_{i}$, given by

$T_{i}=\left[\begin{array}{cc}\left(\mathrm{e}^{\mathrm{2} \mathrm{j} \Gamma_{i} d_{i}}+1\right) & -Z_{i}\left(\mathrm{e}^{2 \mathrm{j} \Gamma_{i} d_{i}}-1\right) \\ \frac{-1}{Z_{i}}\left(\mathrm{e}^{2 \mathrm{j} \Gamma_{i} d_{i}}-1\right) & \left(\mathrm{e}^{2 \mathrm{j} \Gamma_{i} d_{i}}+1\right)\end{array}\right]$

with $Z_{i}=\frac{1}{\Gamma_{i}}$ for TE modes and $Z_{i}=\frac{\Gamma_{i}}{\varepsilon_{i}}$ for TM modes, and with $\Gamma_{i}=\sqrt{\varepsilon_{i}-\beta^{2}}$. In order to determine the number of zeros within a certain contour, the derivative of this matrix with respect to $\beta$ is also required, and is given by

$\frac{\partial T_{i}}{\partial \beta}=\left[\begin{array}{ll}A_{11} & A_{12} \\ A_{21} & A_{22}\end{array}\right]$

with

$A_{11}=\frac{-2 \mathrm{j} \beta d_{i}}{\Gamma_{i}} \mathrm{e}^{2 \mathrm{j} \Gamma_{i} d_{i}}$,

$A_{12}=-\frac{\partial Z_{i}}{\partial \beta}\left(\mathrm{e}^{2 \mathrm{j} \Gamma_{i} d_{i}}-1\right)+\frac{2 \mathrm{j} Z_{i} \beta d_{i}}{\Gamma_{i}} \mathrm{e}^{2 \mathrm{j} \Gamma_{i} d_{i}}$,

$A_{21}=\frac{1}{Z_{i}^{2}} \frac{\partial Z_{i}}{\partial \beta}\left(\mathrm{e}^{2 \mathrm{j} \Gamma_{i} d_{i}}-1\right)+\frac{2 \mathrm{j} \beta d_{i}}{Z_{i} \Gamma_{i}} \mathrm{e}^{2 \mathrm{j} \Gamma_{i} d_{i}}$,

$A_{22}=\frac{-2 \mathrm{j} \beta d_{i}}{\Gamma_{i}} \mathrm{e}^{2 \mathrm{j} \Gamma_{i} d_{i}}$

and with $\frac{\partial Z_{i}}{\partial \beta}=\frac{\beta}{\Gamma_{i}^{3}}$ for TE modes and $\frac{\partial Z_{i}}{\partial \beta}=-\frac{\beta}{\Gamma_{i} \varepsilon_{i}}$ for TM modes. The contribution of the $N-1$ bottom layers to the dispersion relation is then given by $\mathbf{t}_{\text {bottom }}=T_{N-1} \cdot \ldots$. $T_{1} \cdot \mathbf{b}$, with the column vector $\mathbf{b}$ describing the termination. When the bottom layer is terminated by a PEC plate, we have $\mathbf{b}=[0,1]^{\mathrm{T}}$, whereas for an infinite bottom layer $\mathbf{b}=\left[1,-\frac{1}{Z_{0}}\right]^{\mathrm{T}}$. The contribution of the top layer is similar: $\mathbf{t}_{\text {top }}=\left[-1,-\frac{1}{Z_{0}}\right]^{\mathrm{T}}$ for an infinite top layer and $\mathbf{t}_{\text {top }}=$ $\left[Z_{0}\left(\mathrm{e}^{2 \mathrm{j} \Gamma_{0} \tilde{d}_{N}}-1\right),\left(\mathrm{e}^{2 \mathrm{j} \Gamma_{0} \tilde{d}_{N}}+1\right)\right]^{\mathrm{T}}$ when it consists of a PML terminated by a PEC plate, with $\tilde{d}_{N}=d_{N}\left(\kappa-\mathrm{j} \frac{\sigma}{\omega \varepsilon_{0}}\right)(\kappa$ and $\sigma$ being material parameters describing the PML [5]).The 
dispersion relation for the complex propagation constants $\beta$ of the layered medium is therefore of the form $f(\beta)=0$, with

$f(\beta)=\operatorname{det}\left[\mathbf{t}_{\text {bottom, }}-\mathbf{t}_{\text {top }}\right]$.

The derivative is then found as

$f^{\prime}(\beta)=\operatorname{det}\left[\frac{\partial \mathbf{t}_{\text {bottom, }}}{\partial \beta}-\mathbf{t}_{\text {top }}\right]+\operatorname{det}\left[\mathbf{t}_{\text {bottom, }}-\frac{\partial \mathbf{t}_{\text {top }}}{\partial \beta}\right]$

with

$$
\begin{aligned}
& \frac{\partial \mathbf{t}_{\text {bottom }}}{\partial \beta} \\
& \quad=\sum_{i=1}^{N-1} T_{N-1} \cdot \ldots T_{i+1} \cdot \frac{\partial T_{i}}{\partial \beta} \cdot T_{i-1} \cdot \ldots \cdot T_{1} \cdot \mathbf{b} \\
& \quad+T_{N-1} \cdot \ldots \cdot T_{1} \cdot \frac{\partial \mathbf{b}}{\partial \beta} .
\end{aligned}
$$

Let us now focus for a moment on a layered medium terminated by means of a PEC with PML coating. For a single layered microstrip substrate terminated by a PML, it was shown in [27] that, for a high mode number $n$, the modes can be decomposed into leaky modes that depend only on the characteristics of the microstrip and modes that depend mainly on the characteristics of the PML. Following [28], approximate analytical expressions for both modes can then be used as initial estimates, and some simple Newton steps

$\beta \rightarrow \beta-\frac{f(\beta)}{f^{\prime}(\beta)}$

allow to determine all the propagation constants rapidly and accurately. In the general case of $N$ layers, for a high mode number $n$, the modes can still be decomposed into leaky modes that depend only on the characteristics of the $N-1$ bottom layers and modes that depend mainly on the characteristics of the PML. Analytical expressions can be derived for the latter modes for large mode numbers, by making the approximations $\left|\mathrm{e}^{+2 \mathrm{j} \gamma_{r} d}\right| \ll 1$ and $\gamma_{r} \approx \gamma_{0} \approx+\mathrm{j} \beta$. The contribution of the $N-1$ bottom layers is then approximated by $\mathbf{b}=\left[1, \frac{-1}{Z_{\text {eff }}}\right]^{\mathrm{T}}$, i.e. an infinite layer with an effective permittivity that can be calculated analytically. This means that the technique described in [28] can be applied to determine the eigenvalues of these modes rapidly. On the other hand, the other set of modes are approximately given by the leaky modes of the $N-1$ bottom layers, terminated by an open infinite top layer, since for these modes $\left|\mathrm{e}^{-2 \mathrm{j} \gamma_{0}} \tilde{d}\right| \ll 1$ so that

$\mathbf{t}_{\text {top }}=\left[\begin{array}{c}Z_{0}\left(\mathrm{e}^{2 \mathrm{j} \Gamma_{0} \tilde{d}_{N}}-1\right) \\ \left(\mathrm{e}^{2 \mathrm{j} \Gamma_{0} \tilde{d}_{N}}+1\right)\end{array}\right] \approx-Z_{0} \mathrm{e}^{2 \mathrm{j} \Gamma_{0} \tilde{d}_{N}}\left[\begin{array}{c}-1 \\ -1 / Z_{0}\end{array}\right]$.

Since these modes do not depend on the characteristics of the PML anymore, we will not consider PML terminated substrates in the sequel and focus our attention on locating the leaky modes of open layered substrates.

\subsection{Numerical issues}

Before tackling the actual examples, we wish to make the following general remark. Whenever the roots, obtained as a result of the signal identification approach, are found to lack sufficient accuracy, we can terminate the procedure by a few Newton steps (36), as discussed in the previous section, in order to further enhance the global precision defined as

$\mathscr{P}=\max \left(\left|f\left(\beta_{1}\right)\right|,\left|f\left(\beta_{2}\right)\right|, \ldots,\left|f\left(\beta_{m}\right)\right|\right)$.

In all the examples we utilize a 1024 point FFT.

Keep in mind that it is important to restrict the radii of the disks or halfdisks such that there are a limited number of roots in it, say less than 20. The reason for this is that the Hankel moments 'blow up' and lose numerical precision when the radii become too large. Also, the determination of the number of roots by means of the principle of the argument becomes inaccurate when the radii are too large.

\subsection{Example I: a microstrip substrate}

In order to validate the algorithms developed in Section 3 , we consider a planar dielectric microstrip substrate with thickness $d=9 \mathrm{~mm}$ and permittivity $\varepsilon_{r}=3$ at $12 \mathrm{GHz}$. The substrate has no magnetic contrast $\left(\mu_{r}=1\right)$. The propagation constants for this structure were already calculated in [28], based on analytical estimates for the zeros followed by Newton iteration steps.

In Fig. 2, the propagation constants of the leaky modes of this substrate are shown, now found by using the four signal identification algorithms. The conformal mapping technique of Section 4 is used to restrict the domains in which the propagation constants are located to the halfdisks shown in the figure. In order to ensure that none of the zeros is located on the boundary of the halfdisk, we rely on the estimate of the imaginary part of the propagation constants at high mode numbers $n$, given by $-\frac{(2 n+1) \pi}{2 d}$ [28]. We therefore choose the imaginary part of the center of the halfdisks as $\frac{(2 m+1) \pi}{2 d}$ and the radius as $\frac{(2 l+1) \pi}{2 d}$, with $l$ and $m$ integers. The radius is chosen such that about 13 zeros are found in each halfdisk. The halfdisk close to the real axis is chosen smaller, because of the reduced accuracy in this region for zero finding, due to the presence of a branchcut. This smaller halfdisk accommodates seven zeros. The technique is not used to determine the propagation constants of the guided modes, located on the real axis. For these modes, a zero search along the real axis is faster and more accurate, because of the presence of a branchcut. In total 59 propagation constants for the TE modes and 60 propagation constants for the TM modes are found by defining 5 halfdisks. Five Newton steps are used to improve the accuracy of the zeros, whenever necessary. The technique was implemented in MATLAB ${ }^{\mathrm{TM}}$ and the calculations were performed on a 2.4 GHz Pentium IV processor. For the TE modes, algorithm 1 takes $3.20 \mathrm{~s}$ to determine the zeros with a global precision 
Table 1. Number of accurate digits for some normalized propagation constants $\frac{\beta}{k_{0}}$ of TE leaky modes without Newton steps

\begin{tabular}{rllllll}
\hline Mode no. & Estim. [28] & Alg. 1 & Alg. 2 & Alg. 3 & Alg. 4 & Exact zero $\frac{\beta}{k_{0}}$ \\
\hline 4 & 2 & 6 & 6 & 6 & 6 & $0.997134477325266-\mathrm{j} 5.93895922247753$ \\
15 & 4 & 7 & 8 & 8 & 7 & $1.51504618778711-\mathrm{j} 21.42715886211458$ \\
28 & 4 & 8 & 8 & 8 & 8 & $1.78122110319268-\mathrm{j} 39.52559386486341$ \\
41 & 5 & 6 & 7 & 7 & 6 & $1.94651748790590-\mathrm{j} 57.59795588775396$ \\
54 & 5 & 7 & 7 & 7 & 7 & $2.06663919837921-\mathrm{j} 75.66256876746958$ \\
\hline
\end{tabular}

Table 2. Number of accurate digits for some normalized propagation constants $\frac{\beta}{k_{0}}$ of TM leaky modes without Newton steps

\begin{tabular}{rllllll}
\hline Mode no. & Estim. [28] & Alg. 1 & Alg. 2 & Alg. 3 & Alg. 4 & Exact zero $\frac{\beta}{k_{0}}$ \\
\hline 4 & 2 & 7 & 7 & 7 & 7 & $0.16405949006507-\mathrm{j} 6.00562039879446$ \\
15 & 3 & 8 & 8 & 8 & 8 & $0.15407757221948-\mathrm{j} 21.45799596883775$ \\
28 & 4 & 8 & 8 & 8 & 8 & $0.15347207447894-\mathrm{j} 39.54542183237766$ \\
41 & 4 & 8 & 8 & 8 & 8 & $0.15333833987778-\mathrm{j} 57.61285936490915$ \\
54 & 4 & 8 & 8 & 8 & 8 & $0.15328824481330-\mathrm{j} 75.67462553460562$ \\
\hline
\end{tabular}
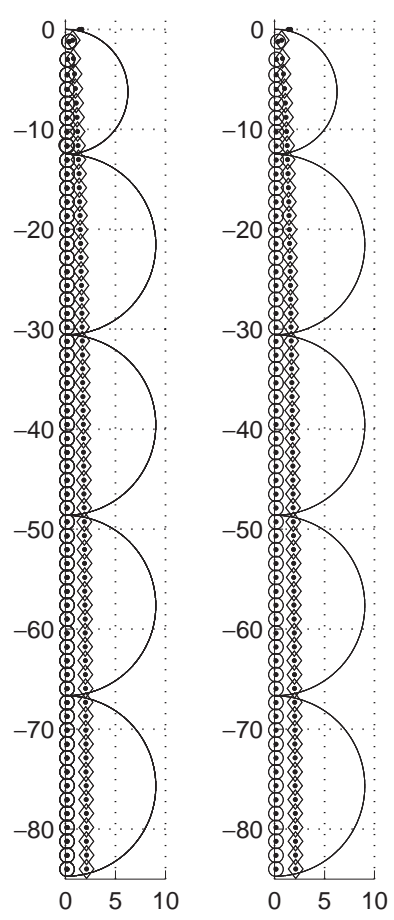

Method 1
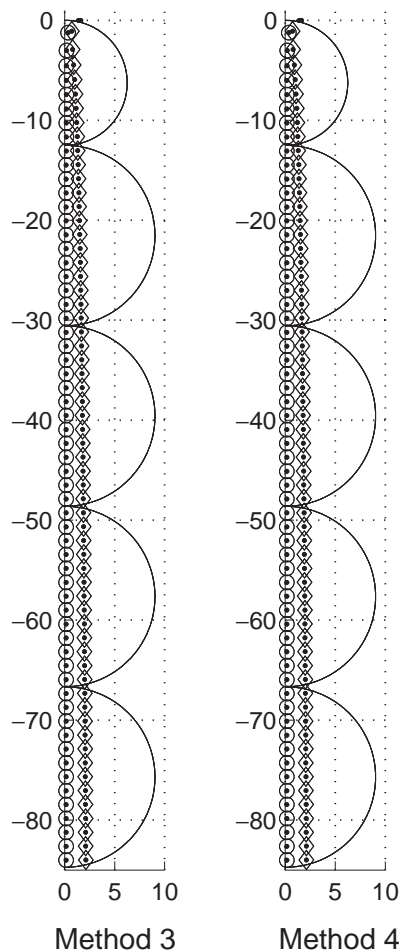

Method 4

Fig. 2. Leaky modes in the microstrip substrate. Circles: TM modes, diamonds: TE modes, points: [28].

$\mathscr{P}=6.45 \times 10^{-16}$, method 2 takes $3.22 \mathrm{~s}$ to determine the zeros with a global precision $\mathscr{P}=5.06 \times 10^{-16}$, technique 3 takes $3.23 \mathrm{~s}$ to determine the zeros with a global precision $\mathscr{P}=5.98 \times 10^{-16}$ and method 4 takes $3.31 \mathrm{~s}$ to determine the zeros with a global precision $\mathscr{P}=6.36 \times 10^{-16}$. For the TM modes, algorithm 1 takes $3.16 \mathrm{~s}$ to determine the zeros with a global precision $\mathscr{P}=2.54 \times 10^{-14}$, method 2 takes $3.16 \mathrm{~s}$ to determine the zeros with a global preci-

sion $\mathscr{P}=5.51 \times 10^{-14}$, algorithm 3 takes $3.17 \mathrm{~s}$ to determine the zeros with a global precision $\mathscr{P}=2.68 \times 10^{-14}$ and method 4 takes $3.26 \mathrm{~s}$ to determine the zeros with a global precision $\mathscr{P}=2.68 \times 10^{-14}$. For the single layer problem, the technique described in [28] is of course faster, requiring $0.328 \mathrm{~s}$ to determine the $59 \mathrm{TE}$ propagation constants with a global precision $\mathscr{P}=2.84 \times 10^{-14}$ and $0.234 \mathrm{~s}$ to determine the $60 \mathrm{TM}$ propagation constants with a global precision $\mathscr{P}=2.84 \times 10^{-14}$. In order to provide more insight into the accuracy of the technique, Table 1 presents the number of accurate digits for some normalized propagation constants $\frac{\beta}{k_{0}}$ of TE leaky modes and Table 2 gives the number of accurate digits for some normalized propagation constants $\frac{\beta}{k_{0}}$ of TM leaky modes, for the analytical estimates in [28] and obtained with the four different algorithms of Section 3, without applying additional Newton steps. The four signal processing techniques provide much more accurate estimates than the analytical expression of Rogier et al. [28]. It is found that the second and the third algorithm provide slightly more accurate zeros than the first and the fourth method.

\subsection{Example II: a two-layered substrate}

Consider a substrate consisting of two dielectric layers, terminated by a PEC plate at the bottom and by an open air region at the top, as shown in Fig. 3. The bottom dielectric has permittivity $\varepsilon_{1}=7.0 \varepsilon_{0}$ and thickness $d_{1}=5 \mathrm{~mm}$, whereas the top dielectric has permittivity $\varepsilon_{2}=3.0 \varepsilon_{0}$ and thickness $d_{2}=4 \mathrm{~mm}$. In Fig. 4 , the propagation constants of the leaky modes of this substrate are shown, found by using the four signal identification algorithms. The halfdisks that were used to locate the zeros are also shown in the figure. To select the halfdisks, we make use of the fact that for high mode 


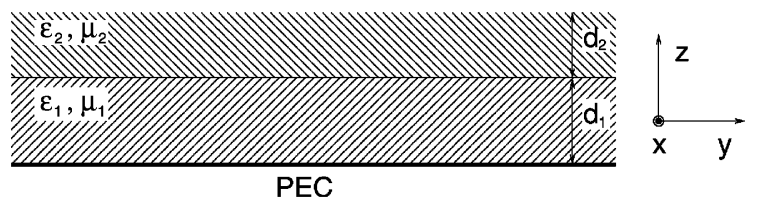

Fig. 3. A substrate consisting of two dielectric layers.
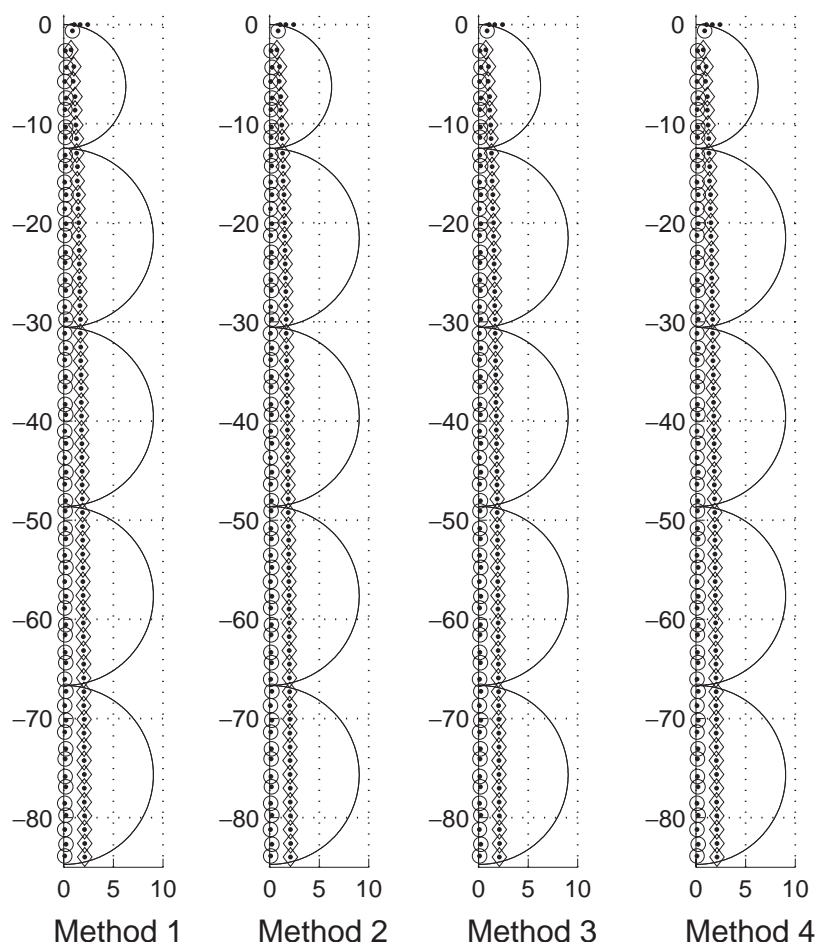

Fig. 4. Leaky modes in the two-layered substrate. Circles: TM modes, diamonds: TE modes, points: principal of the argument method.

numbers $n$, the imaginary part of the zeros is approximately given by $-\frac{(2 n+1) \pi}{2\left(d_{1}+d_{2}\right)}$. We therefore choose the imaginary part of the center of the halfdisks as $\frac{(2 m+1) \pi}{2\left(d_{1}+d_{2}\right)}$ and the radius as $\frac{(2 l+1) \pi}{2\left(d_{1}+d_{2}\right)}$, with $l$ and $m$ integers. The radius is chosen such that about 13 zeros are found in each halfdisk. The halfdisk close to the real axis is again chosen smaller and accommodates seven zeros. In total, 59 propagation constants for the TE modes and 60 propagation constants for the TM modes are found by defining 5 halfdisks. For the TE modes, method 1 takes $5.42 \mathrm{~s}$ to determine the zeros with a global precision $\mathscr{P}=4.4 \times 10^{-15}$, method 2 takes $5.42 \mathrm{~s}$ to determine the zeros with a global precision $\mathscr{P}=8.22 \times 10^{-16}$, method 3 takes $5.44 \mathrm{~s}$ to determine the zeros with a global precision $\mathscr{P}=8.09 \times 10^{-16}$ and method 4 takes $5.47 \mathrm{~s}$ to determine the zeros with a global precision $\mathscr{P}=3.53 \times 10^{-14}$. For the TM modes, method 1 takes $5.38 \mathrm{~s}$ to determine the zeros with a global precision $\mathscr{P}=3.45 \times 10^{-14}$, method 2 takes $5.38 \mathrm{~s}$ to determine the zeros with a global precision $\mathscr{P}=4.38 \times 10^{-14}$, method 3 takes $5.38 \mathrm{~s}$ to determine the zeros with a global precision $\mathscr{P}=3.09 \times 10^{-14}$ and method 4 takes $5.44 \mathrm{~s}$ to determine the zeros with a global precision $\mathscr{P}=7.69 \times 10^{-14}$. All algorithms have comparable CPU times and comparable accuracies, with the Prony-Burrus-Parks method being slightly more accurate. In order to determine the same propagation constants with the principal of the argument method (implemented in $\mathrm{C}$ on a Pentium IV processor), it takes $59 \mathrm{~s}$ for the TE modes and $59 \mathrm{~s}$ for the TM modes.

\section{Acknowledgement}

H. Rogier is a Postdoctoral Fellow of the FWO-Flanders. His research is supported by a grant of the DWTC/SSTC, MOTION project.

\section{Appendix A.}

\section{A.1. Appendix}

The error terms can be written as

$$
\begin{aligned}
\varepsilon_{n}= & \frac{1}{2 \pi} \int_{0}^{2 \pi} \mathrm{e}^{i(n+1) \theta} G(\theta) \mathrm{d} \theta \\
& -\frac{1}{N} \sum_{k=0}^{N-1} \mathrm{e}^{2 \pi i(n+1) k / N} G(2 \pi k / N),
\end{aligned}
$$

where

$G(\theta)=\frac{1}{f\left(\eta\left(\mathrm{e}^{i \theta}\right)\right)}$.

In [29] p. 345, it has been proved that the error $\mathscr{E}(f)$ of the $(N+1)$-point closed trapezoidal rule

$$
\begin{aligned}
\mathscr{E}(f)= & \int_{a}^{b} f(t) \mathrm{d} t-\Delta\left[\frac{f(a)}{2}+\frac{f(b)}{2}\right. \\
& \left.+\sum_{k=1}^{N-1} f(a+k \Delta)\right]
\end{aligned}
$$

with $\Delta=\frac{b-a}{N}$, satisfies the inequality

$|\mathscr{E}(f)| \leqslant \frac{(b-a)^{2}}{4 N}\left[|f(a)|+\sup _{a \leqslant t \leqslant b}\left|f^{\prime}(t)\right|\right]$.

Note that, since the trapezoidal rule is exact for linear functions, the above error bound can be sharpened (replace $f(t)$ with $f(t)-\alpha-\beta(t-a)$ for some $\alpha, \beta)$ to

$|\mathscr{E}(f)| \leqslant \frac{(b-a)^{2}}{4 N} \inf _{\beta} \sup _{a \leqslant t \leqslant b}\left|f^{\prime}(t)-\beta\right|$.

In our case the error bound (A.4) reads as

$\left|\varepsilon_{n}\right| \leqslant \frac{\pi^{2}}{N}\left[|G(0)|+\sup _{0 \leqslant \theta \leqslant 2 \pi}\left|G^{\prime}(\theta)+i(n+1) G(\theta)\right|\right]$. 
The problem with the error bound (A.6) is that, while $G(\theta)$ is continuous and hence bounded, the derivative $G^{\prime}(\theta)$ may be unbounded, especially when using conformal mappings with corner points. Of course, the corner points can be rounded by using the conformal mapping $\eta_{\tau}(u)=\eta(\tau u)$ where $\tau$ is a positive constant slightly smaller than 1 , say $\tau=0.999$.

We now present another bound based on the $L_{2}$ (hence smoothed) norm of derivatives of $G(\theta)$.

Theorem A.1. Let the pth $(p \geqslant 1)$ derivative $G^{(p)}(\theta)$ be in $L_{2}[0,2 \pi]$ and $\frac{n+1}{N} \leqslant \gamma<1$. Then there is a constant $C_{\gamma, p}$ independent of $N, n$ such that $\left|\varepsilon_{n}\right| \leqslant C_{\gamma, p} N^{-p}$.

Proof. Based on an argument similar to the one in [29] p. 293. Consider the Fourier series

$G(\theta)=\sum_{k=-\infty}^{\infty} c_{k} \mathrm{e}^{i k \theta}$.

By the premises we have

$$
\begin{aligned}
\left\|G^{(p)}\right\|_{2}^{2} & =\frac{1}{2 \pi} \int_{0}^{2 \pi}\left|G^{(p)}(\theta)\right|^{2} \mathrm{~d} \theta \\
& =\sum_{k=-\infty}^{\infty}|k|^{2 p}\left|c_{k}\right|^{2}<\infty .
\end{aligned}
$$

The errors $\varepsilon_{n}$ on the other hand, can be written as

$$
\varepsilon_{n}=c_{-n-1}-\sum_{k=-\infty}^{\infty} c_{k N-n-1}=-\sum_{k \neq 0} c_{k N-n-1} .
$$

Hence

$$
\begin{aligned}
\left|\varepsilon_{n}\right| \leqslant & \left|\sum_{k \neq 0} c_{k N-n-1}\right| \\
\leqslant & \sqrt{\sum_{k \neq 0}|k N-n-1|^{-2 p}} \\
& \times \sqrt{\sum_{k \neq 0}\left|c_{k N-n-1}\right|^{2}|k N-n-1|^{2 p}} \\
\leqslant & N^{-p}\left\|G^{(p)}\right\|_{2} \sqrt{\sum_{k \neq 0}\left|k-\frac{n+1}{N}\right|^{-2 p}}
\end{aligned}
$$

by Schwarz inequality. Now putting $\delta=\frac{n+1}{N} \leqslant \gamma<1$, and with $\zeta(\cdot)$ the Riemann zeta function, we obtain

$$
\begin{aligned}
\sum_{k \neq 0}|k-\delta|^{-2 p} & \leqslant \sum_{k=1}^{\infty}(k-\delta)^{-2 p}+\zeta(2 p) \\
& \leqslant \zeta(2 p)+\frac{2 p-\delta}{2 p-1}(1-\delta)^{-2 p} \\
& \leqslant \zeta(2 p)+\frac{p}{p-1 / 2}(1-\gamma)^{-2 p}
\end{aligned}
$$

Hence taking

$C_{\gamma, p}=\left\|G^{(p)}\right\|_{2} \sqrt{\zeta(2 p)+\frac{p}{p-1 / 2}(1-\gamma)^{-2 p}}$

completes the proof.

The above theorem, shows that the error terms $\varepsilon_{n}$ are uniformly $O\left(N^{-p}\right)$ provided the relative ratio $\gamma=\frac{n+1}{N}$, which is related to the Hankel moments needed, remains sufficiently small.

\section{References}

[1] Tornero J, Melcon A. Leaky and surface waves in multi-layered laterally-shielded microstrip transmission lines. Microwave Opt Technol Lett 2003;37:88-93.

[2] Baccarelli P, Burghignoli P, Frezza F, Galli A, Lovat G, Jackson D. Approximate analytical evaluation of the continuous spectrum in a substrate-superstrate dielectric waveguide. IEEE Trans Microwave Theory Tech 2002; 50:2690-701.

[3] Nallo CD, Mesa F, Jackson D. Excitation of leaky modes on multilayer stripline structures. IEEE Trans Microwave Theory Tech 1998;46:1062-71.

[4] Chen P, Lee C, Nalbandian V. Planar double-layer leakywave microstrip antenna. IEEE Trans Antennas Propag 2002;50:832-5.

[5] Rogier H, De Zutter D. Convergence behavior and acceleration of the Berenger and leaky modes series composing the 2D Green's function for the microstrip substrate. IEEE Trans Microwave Theory Tech 2002;50:1696-704.

[6] Rogier H, De Zutter D. A fast technique based on perfectly matched layers to model electromagnetic scattering from wires embedded in substrates. Radio Sci 2001;37:10.1-6.

[7] Rogier H, De Zutter D. A fast technique based on perfectly matched layers for the full-wave space domain solution of 2D dispersive microstrip lines. IEEE Trans CAD Integrated Circuits Systems 2003;22:1650-6.

[8] Holmström K, Petersson J. A review of the parameter estimation problem of fitting positive exponential sums to empirical data. Appl Math Comput 2002;126:31-61.

[9] Kung SY, Arun KS, Rao DVB. State-space and singularvalue decomposition-based approximation methods for the harmonic retrieval problem. J Opt Soc Am 1983;73:1799811.

[10] Redheffer RM. Completeness of sets of complex exponentials. Adv Math 1977;24:1-62.

[11] Knockaert L. Equivalent formulations of the Müntz-Szász completeness condition for systems of complex exponentials. J Franklin Inst 2002;339:103-9.

[12] Gragg WB, Lindquist A. On the partial realization problem. Linear Algebra Appl 1983;50:277-319.

[13] Boley DL, Luk FT, Vandevoorde D. A fast method to diagonalize a hankel matrix. Linear Algebra Appl 1998;284:41-52.

[14] Tamm U. Some aspects of hankel matrices in coding theory and combinatorics. Electron J Combin 2001;8:A1:1-31. 
[15] Golub GH, Milanfar P, Varah J. A stable numerical method for inverting shape from moments. SIAM J Sci Comput 1999;21:1222-43.

[16] Luck R, Stevens JW. Explicit solutions for transcendental equations. SIAM Rev 2002;44:227-33.

[17] Kravanja P, Van Barel M. Computing the zeros of analytic functions. Lecture Notes Math 2000;1727:1-59.

[18] Hua Y, Sarkar TK. Matrix pencil method for estimating parameters of exponentially damped/undamped sinusoids in noise. IEEE Trans Acoust Speech Signal Process 1990;38: 814-24.

[19] Burrus CS, Parks TW. Time domain design of recursive digital filters. IEEE Trans Audio Electroacoustics 1970;AU-18: 137-41.

[20] Krauss TP, Shure L, Little JN. Signal processing toolbox for use with matlab: user's guide. Natick: The MathWorks; 1994.

[21] Lehmensiek R, Meyer P. An efficient adaptive frequency sampling algorithm for model-based parameter estimation as applied to aggressive space mapping. Microwave Opt Technol Lett 2000;24:71-8.

[22] Henrici P. Applied and computational complex analysis, vol. 1. New York: Wiley Classics; 1988.

[23] Henrici P. Applied and computational complex analysis, vol. 3. New York: Wiley Classics; 1988.

[24] Cadzow JA. Signal enhancement-a composite property mapping algorithm. IEEE Trans Acoust Speech Signal Process 1988;36:49-62.

[25] Michalski KA, Mosig JR. Multilayer media Green's functions in integral equation formulations. IEEE Trans Antennas Propag 1997;45:508-19.

[26] Faché N, Van Hese J, De Zutter D. Generalised space domain Green's dyadic for multilayered media with special application to microwave interconnections. J Electromagnetic Waves Appl 1992;3:651-69.

[27] Rogier H, De Zutter D. Berenger and leaky modes in microstrip substrates terminated by a perfectly matched layer. IEEE Trans Microwave Theory Tech 2001;49:712-5.

[28] Rogier H, Knockaert L, De Zutter D. Fast calculation of the propagation constants of leaky and Berenger modes of planar and circular dielectric waveguides terminated by a perfectly matched layer. Microwave Opt Technol Lett 2003;37:167-71.

[29] Davis PJ. Interpolation \& approximation. New York: Wiley Classics; 1975.

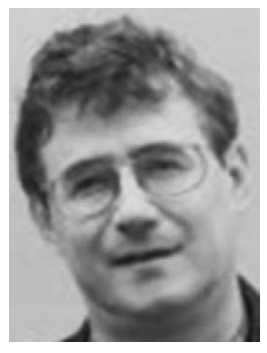

Luc Knockaert received the M.Sc. Degree in Physical Engineering, M.Sc. Degree in Telecommunications Engineering and Ph.D. Degree in Electrical Engineering from Ghent University, Belgium, in 1974, 1977, and 1987, respectively.

From 1979 to 1984 and from 1988 to 1995 he was working in North-South cooperation and development projects at the Universities of Congo (formerly Zaire) and Burundi. He is presently a guest professor and senior researcher at INTEC-IMEC. His current interests are the application of statistical and linear algebra methods in signal identification, entropy applications, matrix compression, reduced order modelling and computational electromagnetics. As author or coauthor he has contributed to more than 40 international ISI Web of Science peer-reviewed journal papers.

Dr. Knockaert is a member of ACM, SIAM and a senior member of IEEE.

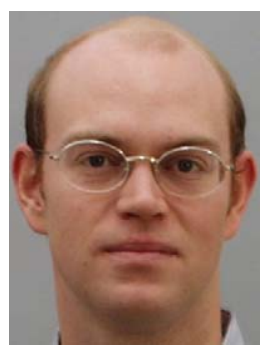

Hendrik Rogier was born in 1971. He received the Electrical Engineering degree and the Ph.D. Degree from Ghent University, Ghent, Belgium, in 1994 and in 1999, respectively.

$\mathrm{He}$ is currently a Postdoctoral Fellow of the FWO-Flanders and Assistant Professor in mobile communications at the Department of Information Technology of Ghent University. He authored and co-authored more than 20 papers in international journals and more than 25 contributions in conference proceedings. He has one patent pending. His current research interests are the analysis of electromagnetic waveguides, electromagnetic simulation techniques applied to EMC and signal integrity problems, as well as to indoor propagation and antenna design, and in smart antenna systems for wireless networks.

Dr. Rogier received the URSI Young Scientist Award at the 2001 URSI Symposium on Electromagnetic Theory and at the 2002 URSI General Assembly.

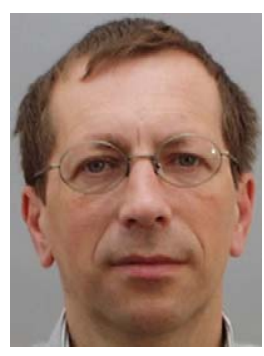

Daniël De Zutter was born in 1953. He received his M.Sc. Degree in Electrical Engineering from Ghent University in 1976. From 1976 to 1984 he was a Research and Teaching Assistant at the same university. In 1981 he obtained a Ph.D. degree and in 1984 he completed a thesis leading to a degree equivalent to the French Aggrégation or the German Habilitation. From 1984 to 1996 he was with the National Fund for Scientific Research of Belgium. He is now a full professor of electromagnetics. Most of his earlier scientific work dealt with the electrodynamics of moving media. His research now focusses on all aspects of circuit and electromagnetic modelling of high-speed and high-frequency interconnections and packaging, on Electromagnetic Compatibility (EMC) and numerical solutions of Maxwell's equations. As author or co-author he has contributed to more than 110 international journal papers and 140 papers in conference proceedings. In 1993 he published a book titled "Electromagnetic and circuit modelling of multiconductor transmission lines" (with $\mathrm{N}$. Faché and F. Olyslager) in the Oxford Engineering Science Series. He received the 1990 Montefiore Prize of the University of Liège and the 1995 IEEE Microwave Prize Award (with F. Olyslager and K. Blomme) from the IEEE Microwave Theory and Techniques Society for best publication in the field of microwaves for the year 1993. In 1990, he was elected as a Member of the Electromagnetics Society. In 1999, he received the Transactions Prize Paper Award from the IEEE EMC Society. In 2000, he was elected to the grade of Fellow of the IEEE. 